\title{
Injury Evaluation of Human-Robot Impacts
}

\author{
Sami Haddadin, Alin Albu-Schäffer, Michael Strohmayr, Mirko Frommberger, and Gerd Hirzinger
}

\begin{abstract}
Currently, large efforts are unertaken to bring robotic applications to domestic environments. Especially physical human-robot cooperation is a major concern and various design and control methodologies were developed on the way to achieve this task. In particular, this necessitates the evaluation of injury risks a human is exposed to in case he is hit by a robot. In this video several blunt impact tests are shown, leading to an assessment of which factors dominate injury severity. We will illustrate the effect robot speed, robot mass, and constraints in the environment have on safety in humanrobot impacts. It will be shown that the intuition of high impact loads being transmitted by heavy robots is wrong. Furthermore, the conclusion is induced that free impacts are by far less dangerous than being crushed.
\end{abstract}

\section{Motivation \& InTRODUCTION}

Up to now injuries occurring in human-robot impacts have not been sufficiently studied. Various questions arise, especially regarding the major factors defining resulting injury. In this video the effect of robot mass, robot speed, and constraints in the environment on injury severity during human-robot impacts are explained and supported by various crash-tests. Although there is some literature present treating safety issues in human-robot interaction [1], [2], [3], [4], [5], there was so far no effort taken to analyze real world threats via impact tests at standardized crash-test facilities. This was to our knowledge only carried out in [6] up to now.

\section{Test SETUP}

In [6] results and implications from impact tests at certified crash-test facilities with the DLR Lightweight Robot III (LWRIII) were obtained, see Fig. 1a. Because such crashtests are very expensive ${ }^{1}$, we decided to use the resulting outcome of the impact tests to built up a simplified setup that mimics a HybridIII dummy head and use it for the evaluation of other robots.

These results indicate that a robot, even with arbitrary mass driving not much faster than $2 \mathrm{~m} / \mathrm{s}$ is not able to become dangerous to a non-clamped human head with respect to typical severity indices ${ }^{2}$ used in automobile industry, usually focusing on head acceleration. In this video we confirm this strong statement by evaluating the Head Injury Criterion

S. Haddadin, A. Albu-Schäffer, Michael Strohmayr, Mirko Frommberger and G. Hirzinger are with Institute of Robotics and Mechatronics, DLR - German Aerospace Center, Wessling, Germany sami.haddadin, alin.albu-schaeffer, michael.strohmayr, mirko.frommberger gerd.hirzingerdalr.de

This work has been partially funded by the European Commission's Sixth Framework Programme as part of the projects SMERobot ${ }^{\text {TM }}$ under grant no. 011838 and PHRIENDS under grant no. 045359.

${ }^{1} \mathrm{Up}$ to 1000 Euros per impact. For an entire test series at various impact velocities this would be a very high expense.

${ }^{2}$ These are established measures of injury severity which are usually defined for certain body regions. a.

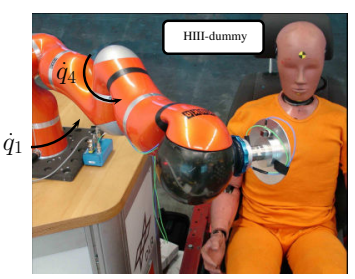

b.

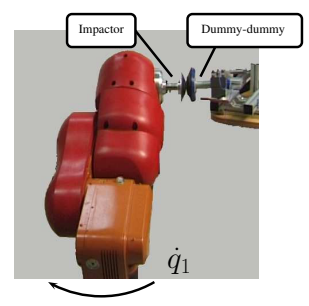

c.

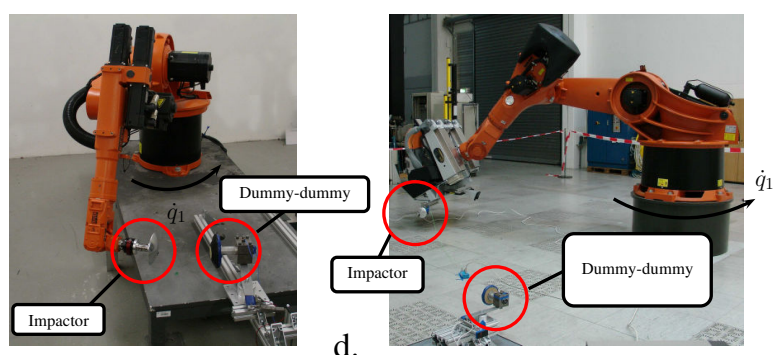

Fig. 1. Setup of impact tests with the DLR-LWRIII (a.), KUKA KR3SI (b.), KUKA KR6 (c.) and KUKA KR500 (d.). Reflected inertias in the direction of impact were $\{4,12,67,1870\} \mathrm{kg}$.

(HIC) [7], defined as

$\mathrm{HIC}_{36}=\max _{(\Delta t)}\left\{(\Delta t)\left(\frac{1}{\Delta t} \int_{t_{1}}^{t_{2}}\left\|\ddot{\mathbf{x}}_{H}\right\|_{2} \mathrm{~d} t\right)^{\left(\frac{5}{2}\right)}\right\} \leq 1000$
$\Delta t=t_{2}-t_{1} \leq \Delta t_{\max }=36 \mathrm{~ms}$.

$\left\|\ddot{\mathbf{x}}_{H}\right\|$ is the resulting acceleration of the human head ${ }^{3}$ and has to be measured in $\mathrm{g}=9.81 \mathrm{~m} / \mathrm{s}^{2}$. Its correlation to injury severity can be obtained from Fig. 2. A numerical value of $\leq 650$ corresponds to very low injury by means of the EuroNCAP ${ }^{4}$. For further information on HIC, AIS and other Severity Indices (not only for the head), please refer to [6].

In order to cover a wide range of robots and be able to verify the saturation effect explained in [6], we compare the LWRIII with typical industrial robots ${ }^{5}$ of different weight, see Fig. 1.

\section{HEAD INJURY CRITERION}

In this video, impact experiments with various robots are shown and the resulting HIC values are indicated. These values are also summarized in Fig. 2. They are additionally classified with respect to the EuroNCAP. Clearly, the saturation effect mentioned in Sec. II was observed, as the

\footnotetext{
${ }^{3}\|\ddot{\mathbf{x}}\|_{2}=$ Euclidean norm

${ }^{4}$ The initial crash-tests with the LWRIII were carried out at the German Automobile Club ADAC. They are the basis for the tests presented in this video and are evaluated according to the EuroNCAP. This is a manufacturer independent crash-test program, based on the Abbreviated Injury Scale (AIS). For further information on these issues please refer to [6].

${ }^{5}$ KUKA KR3-SI $(54 \mathrm{~kg})$, the KUKA KR6 $(235 \mathrm{~kg})$, and the KUKA KR500 (2350 kg)
} 


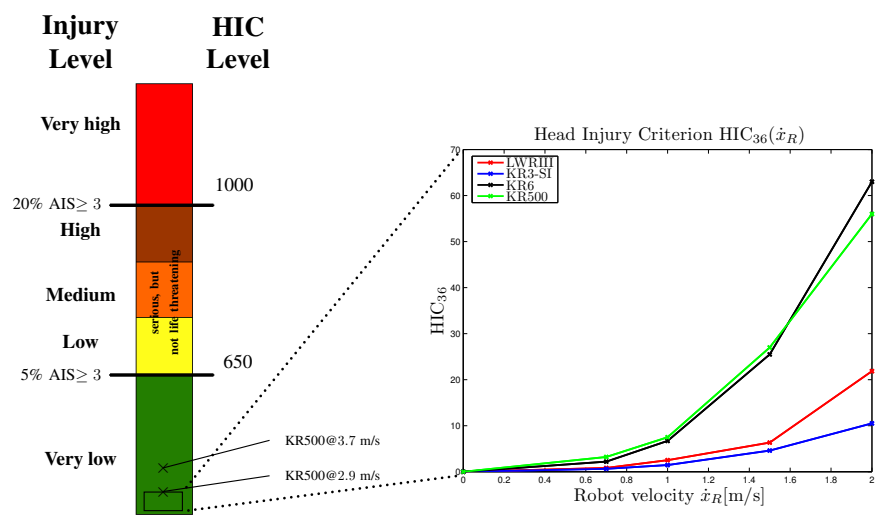

Fig. 2. Resulting $\mathrm{HIC}_{36}$ values for varying impact velocities and for all robots, rated according to the EuroNCAP Assessment Protocol And Biomechanical Limits.

numerical values for the $235 \mathrm{~kg}$ or $2350 \mathrm{~kg}$ robot do not significantly differ. Even at $80 \%$ and $100 \%$ of the maximum joint velocity $\dot{q}_{1}^{\max }$, corresponding to a Cartesian velocity of $2.9 \mathrm{~m} / \mathrm{s}$ and $3.7 \mathrm{~m} / \mathrm{s}$, the measured HIC for the $2350 \mathrm{~kg}$ robot was 135 and 246. This means that even such an enormous robot as the KR500 cannot pose a significant threat by means of impact to the human head measured by typical severity indices from automobile crash-testing ${ }^{6}$, see Fig. 2 . The injury level for these values are located in the green area (see Fig. 2) and the probability of AIS $\geq 3$-injuries are $1.2 \%$ and $3.6 \%$ for the faster impacts with the KR500. A full evaluation of the impact tests, their consequences and further remarks can be found in [8].

\section{SOCCER KICK}

In order to show that an impact cannot be a priori life threatening $^{7}$, a soccer ball was kicked with the $2350 \mathrm{~kg}$ KUKA KR500 at maximum joint velocity. The ball hits the ground after a flight of only $2 \mathrm{~m}$. In comparison, a human performed a kick as well and one can clearly see how slow and careful he hits the ball in order not to shoot farther. Additionally, a rather hard shot was taken to point out the dramatic contrast to the robot. This example clearly gives a better feeling what it means to be hit by the robot at such a velocity. It shows that the intuition of high impacts being transmitted by heavy robots is wrong.

\section{Clamping}

After this impact analysis leading to the conclusion that no robot is able to cause life-threatening injury by means of HIC, we show the effect of the robot mass if the human gets clamped in a constrained environment. This effect is visualized by cracking with the $235 \mathrm{~kg}$ robot KR6 a coconut, which has approximately the same fracture force limit as the human head ${ }^{8}$, see Fig. 4. In this situation the long braking distances and high motor torques can lead to deadly injuries, inducing the conclusion that free impacts are by far less dangerous than being crushed. A detailed analysis on this topic is presented in [9].

\footnotetext{
${ }^{6}$ Please note these statements are made for blunt impacts and not for injuries caused by sharp tools or edges.

${ }^{7}$ Of course, pathological cases cannot be excluded.

${ }^{8}$ In particular, the frontal bone.
}

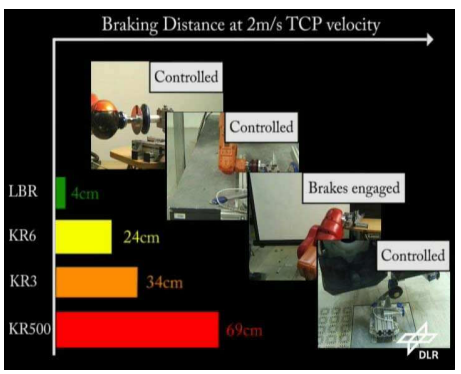

Fig. 3. Comparing the braking distance of robots with different weights.
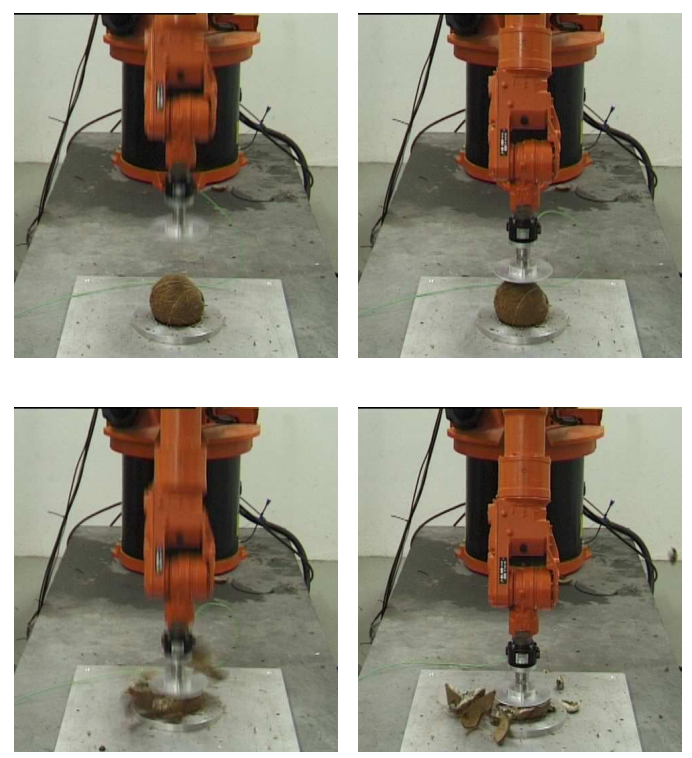

Fig. 4. Cracking a coconut with a KR6.

\section{REFERENCES}

[1] A. Bicchi and G. Tonietti, "Fast and Soft Arm Tactics: Dealing with the Safety-Performance Trade-Off in Robot Arms Design and Control," IEEE Robotics and Automation Mag., vol. 11, pp. 22-33, 2004.

[2] M. Zinn, O. Khatib, and B. Roth, "A New Actuation Approach for Human Friendly Robot Design," Int. J. of Robotics Research, vol. 23, pp. 379-398, 2004.

[3] K. Ikuta, H. Ishii, and M. Nokata, "Safety Evaluation Method of Design and Control for Human-Care Robots," Int. J. of Robotics Research, vol. 22, no. 5, pp. 281-298, 2003.

[4] J. Heinzmann and A. Zelinsky, "Quantitative Safety Guarantees for Physical Human-Robot Interaction," Int. J. of Robotics Research, vol. 22 , no. $7-8$, pp. $479-504,2003$.

[5] H.-O. Lim and K. Tanie, "Human Safety Mechanisms of HumanFriendly Robots: Passive Viscoelastic Trunk and Passively Movable Base," Int. J. of Robotics Research, vol. 19, no. 4, pp. 307-335, 2000.

[6] S. Haddadin, A. Albu-Schäffer, and G. Hirzinger, "Safety Evaluation of Physical Human-Robot Interaction via Crash-Testing," in Robotics: Science and Systems Conference (RSS2007), Atlanta, USA, 2007.

[7] J. Versace, "A Review of the Severity Index," Proc 15th Stapp Conference, vol. SAE Paper No.710881, 1971.

[8] S. Haddadin, A. Albu-Schäffer, and G. Hirzinger, "The Role of the Robot Mass and Velocity in Physical Human-Robot Interaction - Part I: Unconstrained Blunt Impacts," in IEEE Int. Conf. on Robotics and Automation (ICRA2008), Pasadena, USA, 2008.

[9] — , "The Role of the Robot Mass and Velocity in Physical HumanRobot Interaction - Part II: Constrained Blunt Impacts," in IEEE Int. Conf. on Robotics and Automation 2008 (ICRA 2008), Pasadena, USA, 2008. 